\title{
The jet in the galactic center: An ideal laboratory for magnetohydrodynamics and general relativity
}

\author{
Heino Falcke ${ }^{1,2}$, Sera Markoff ${ }^{3}$, Geoffrey C. Bower ${ }^{4}$, Charles F. \\ Gammie $^{5,6}$, Monika Mościbrodzka ${ }^{5}$, and Dipankar Maitra ${ }^{7}$ \\ ${ }^{1}$ Department of Astrophysics, Institute for Mathematics, Astrophysics and Particle Physics \\ (IMAPP), Radboud University, Nijmegen, The Netherlands \\ ${ }^{2}$ ASTRON, Oude Hoogeveensedijk 4, 7991 PD Dwingeloo, The Netherlands \\ ${ }^{3}$ Astronomical Institute "Anton Pannekoek", University of Amsterdam, The Netherlands \\ ${ }^{4}$ Astronomy Department \& Radio Astronomy Lab, UC Berkeley, USA \\ ${ }^{5}$ Department of Physics, University of Illinois, Urbana, Illinois, USA \\ ${ }^{6}$ Astronomy Department, University of Illinois, Urbana, Illinois, USA \\ ${ }^{7}$ Department of Astronomy, University of Michigan, Ann Arbor, Michigan, USA
}

\begin{abstract}
Of all possible black hole sources, the event horizon of the Galactic Center black hole, Sgr A*, subtends the largest angular scale on the sky. It is therefore a prime candidate to study and image plasma processes in strong gravity and it even allows imaging of the shadow cast by the event horizon. Recent mm-wave VLBI and radio timing observations as well as numerical GRMHD simulations now have provided several breakthroughs that put Sgr A* back into the focus. Firstly, VLBI observations have now measured the intrinsic size of Sgr A* at multiple frequencies, where the highest frequency measurements have approached the scale of the black hole shadow. Moreover, measurements of the radio variability show a clear time lag between 22 $\mathrm{GHz}$ and $43 \mathrm{GHz}$. The combination of size and timing measurements, allows one to actually measure the flow speed and direction of magnetized plasma at some tens of Schwarzschild radii. This data strongly support a moderately relativistic outflow, consistent with an accelerating jet model. This is compared to recent GRMHD simulation that show the presence of a moderately relativistic outflow coupled to an accretion flow Sgr A*. Further VLBI and timing observations coupled to simulations have the potential to map out the velocity profile from 5-40 Schwarzschild radii and to provide a first glimpse at the appearance of a jet-disk system near the event horizon. Future submm-VLBI experiments would even be able to directly image those processes in strong gravity and directly confirm the presence of an event horizon.
\end{abstract}

Keywords. accretion, black hole physics, gravitation, MHD, relativity, instrumentation: interferometers, instrumentation: high angular resolution, Galaxy: center, Galaxy: nucleus, galaxies: jets, quasars: general

\section{Introduction}

Based on an analogy to other active galactic nuclei (AGN) Lynden-Bell \& Rees (1971) proposed to look for a compact radio source in the center of our own Milky Way, which was then discovered by Balick \& Brown (1974) only a little later with the NRAO interferometer at Green Bank - barely beating the team at Westerbork (Ekers et al. 1975). This "compact radio source in the Galactic Center" became later known as "Sagittarius $\mathrm{A}^{*}$ " (Sgr A*) and by now is the best constrained super-massive black hole candidate we know of to date (see Melia \& Falcke 2001; Genzel et al. 2010, for a review), with a mass of $4 \times 10^{6} M_{\odot}$ measured from stellar orbits (Schödel et al. 2002; Ghez et al. 2008; Gillessen 
et al. 2009). Sgr A* serves as an excellent example to understand black holes (and compact radio cores) at very low accretion rates and is a potentially exciting laboratory for the study of General Relativity (GR) itself. In fact, Falcke et al. (2000) argued that, what they called the "shadow of the black hole event horizon" could reasonably be detected with mm-wave very long baseline interferometry (VLBI) in this source and many papers have since illuminated various general relativistic effects that could be tested in this way (Broderick \& Loeb 2006; Fish et al. 2009; Harko et al. 2009; Yuan et al. 2009; Johannsen \& Psaltis 2010; Dexter et al. 2010).

However, ever since its discovery, a discussion raged about the exact nature of its emission processes and the region where the observable radiation is emitted. Already Reynolds \& McKee (1980) suggested an origin of the radio emission in a jet or wind from a stellar-sized object. Indeed, the properties of Sgr A* resemble those of flat-spectrum compact radio cores in quasars (later also found in X-ray binaries), which were explained by Blandford \& Königl (1979) as the $\tau=1$ surfaces (optical depth becomes unity) of powerful relativistic jets. This issue was revisited by Falcke et al. (1993); Falcke \& Markoff (2000) who explained spectrum and size of Sgr A* as a scaled-down quasar jet from an "AGN on a starvation diet", i.e. a supermassive black hole with very low accretion rate $\left(\dot{M}<10^{-7} M_{\odot} / \mathrm{yr}\right)$. This semi-analytic approach was based based upon a modified Blandford-Königl model (Falcke 1996, taking the longitudinal pressure gradient into account) and the jet-disk symbiosis ansatz (Falcke \& Biermann 1995, 1999), which introduced a very simple, yet until to day very effective linear scaling between jet-power and accretion disk rate for black holes $Q_{\text {jet }}=q_{\mathrm{j}} \dot{M}_{\text {disk }}$, where $q_{\mathrm{j}} \sim 3-10 \%$. Based on the jet-disk symbiosis model, a number of concrete predictions were made (Falcke 1999a) that all have stood the test of time.

Alternatively, it was proposed that the radio emission - and subsequently the emission at other wavelengths - was produced in the accretion flow itself. While standard optically thick, geometrically thin accretion models started to fail in explaining the low infrared flux of Sgr A* (Falcke \& Melia 1997; Coker et al. 1999), the picture of an optically thin, geometrically thick accretion flow, so-called advection-dominated or radiativelyinefficient accretion flows (ADAFs, RIAFs) started to emerge (Melia 1992; Narayan et al. 1998; Quataert \& Gruzinov 2000). These models initially had much higher accretion rates, but this was brought down when polarization measurements, using Faraday rotation arguments, (Bower et al. 1999; Aitken et al. 2000; Bower et al. 2005; Marrone et al. 2007) showed a low particle density towards Sgr A*. This implied an accretion rate below $10^{-7} M_{\odot} /$ yr. Hence, our Galactic Center is likely starving as well as radiating inefficiently and producing a jet (Yuan et al. 2002).

\section{Review of observational progress}

In recent years, important progress has come from the detection of Sgr A* in X-rays and near-infrared (Baganoff et al. 2001; Genzel et al. 2003) and a lot of emphasis has been placed on variability studies of Sgr A* (Herrnstein et al. 2004; Falcke 1999b; Porquet et al. 2008; Mauerhan et al. 2005; Yusef-Zadeh et al. 2006; Marrone et al. 2008; Eckart et al. 2008; Meyer et al. 2008; Yusef-Zadeh et al. 2009; Dodds-Eden et al. 2009, to name just a few). The various campaigns have shown that flares in the near-infrared (NIR) and $\mathrm{X}$-rays are simultaneous within minutes and hence come from the same region. DoddsEden et al. (2009) favor optically thin synchrotron radiation over inverse Compton as the dominant emission process to explain the remarkable similarity of a particularly bright flare in both bands. On the other hand radio/submm-wave and X-ray/NIR flares do not 

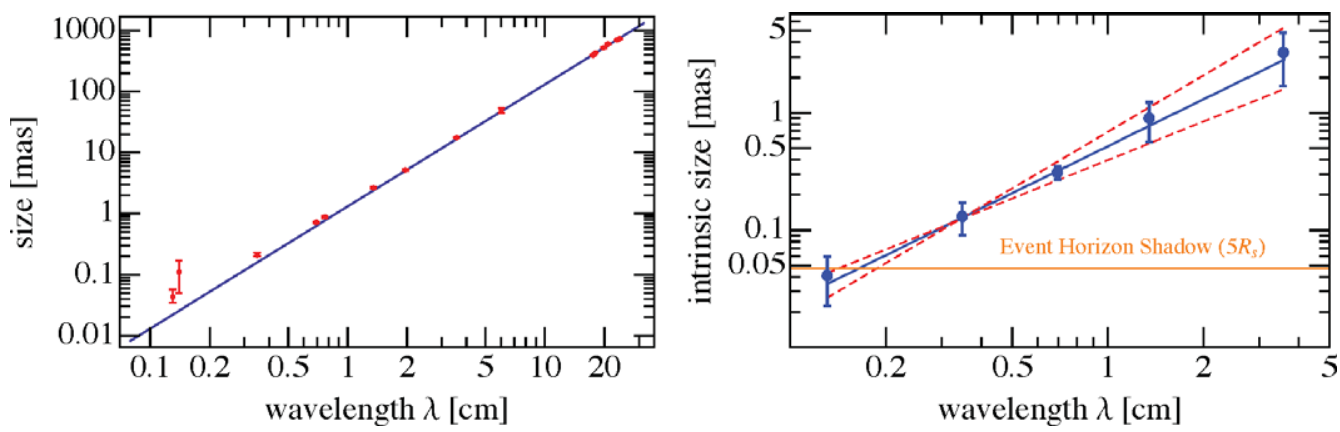

Figure 1. Left: measured major axis size of Sgr A* as function of wavelength measured by various VLBI experiments. Right: Derived intrinsic size of Sgr A* after subtraction of a $\lambda^{2}$ scattering law using all available VLBI data (see Falcke et al. (2009) for details). The dashed line indicates the systematic uncertainties due to different normalizations of the scattering law.

seem to be simultaneous and no generally accepted lag has been derived, despite various claims in the literature, which are ranging from several hours to minutes.

Very exciting has also been the progress based on radio observations, constraining any model - and in particular any jet model - much better. Bower et al. (2004) were the first to measure the intrinsic source size of Sgr A* at $43 \mathrm{GHz}$ directly using Very Long Baseline Interferometry (VLBI), yielding a size of some 20 Schwarzschild radii $\left(R_{\mathrm{S}}\right)$ only. This was a true breakthrough, given that the structure of Sgr A* had been washed out due to interstellar scattering for 30 years since its discovery (yielding a measured size that decreases with $\lambda^{2}$ at low frequencies, Fig. 1, left). Further VLBI measurements then provided measurements up to $220 \mathrm{GHz}$ (Shen et al. 2005; Doeleman et al. 2008) giving sizes down to $4 R_{\mathrm{s}}$ ! Hence, it is clear now that the intrinsic source size decreases with increasing frequency (Fig. 1, right) - an important prediction of the jet model. Direct comparison of the jet model with the actual VLBI data shows a consistency with the compact source size and structure, but does favor edge-on geometries (Markoff et al. 2007).

Additional information has come from the measurement of time lags in the radio (Yusef-Zadeh et al. 2006, 2008), where it was shown that $43 \mathrm{GHz}$ radio flares precede 22 $\mathrm{GHz}$ flares by about 20 minutes. Given that the intrinsic size difference between these two frequencies is about 30 light minutes one arrives at the natural conclusion that the radio emitting plasma flows out with the speed of light (Falcke et al. 2009), which is now also suggested by the short-time variability of the source (Yusef-Zadeh et al. 2009).

Alternative models based on simple adiabatic expansion of "blobs" which expand subrelativisitically (Yusef-Zadeh et al. 2006; Eckart et al. 2009) have only been fitted to the light curves and fail to take the VLBI sizes into account. They predict much smaller source sizes and would postulate two very different emission regions, while fits for adiabatic expansion in a jet (Maitra et al. 2009) do reproduce radio light curves and VLBI sizes very well.

In the following we will now focus on the the basic properties of emission from a jet and briefly discuss how future radio observations and modeling will provide us with deeper insight into the formation of jets close to the event horizon.

\section{The basic jet model}

Given the observational progress any model for Sgr A* nowadays has to explain the spectrum, size, and variability properties of the source. Here we will briefly explain the 


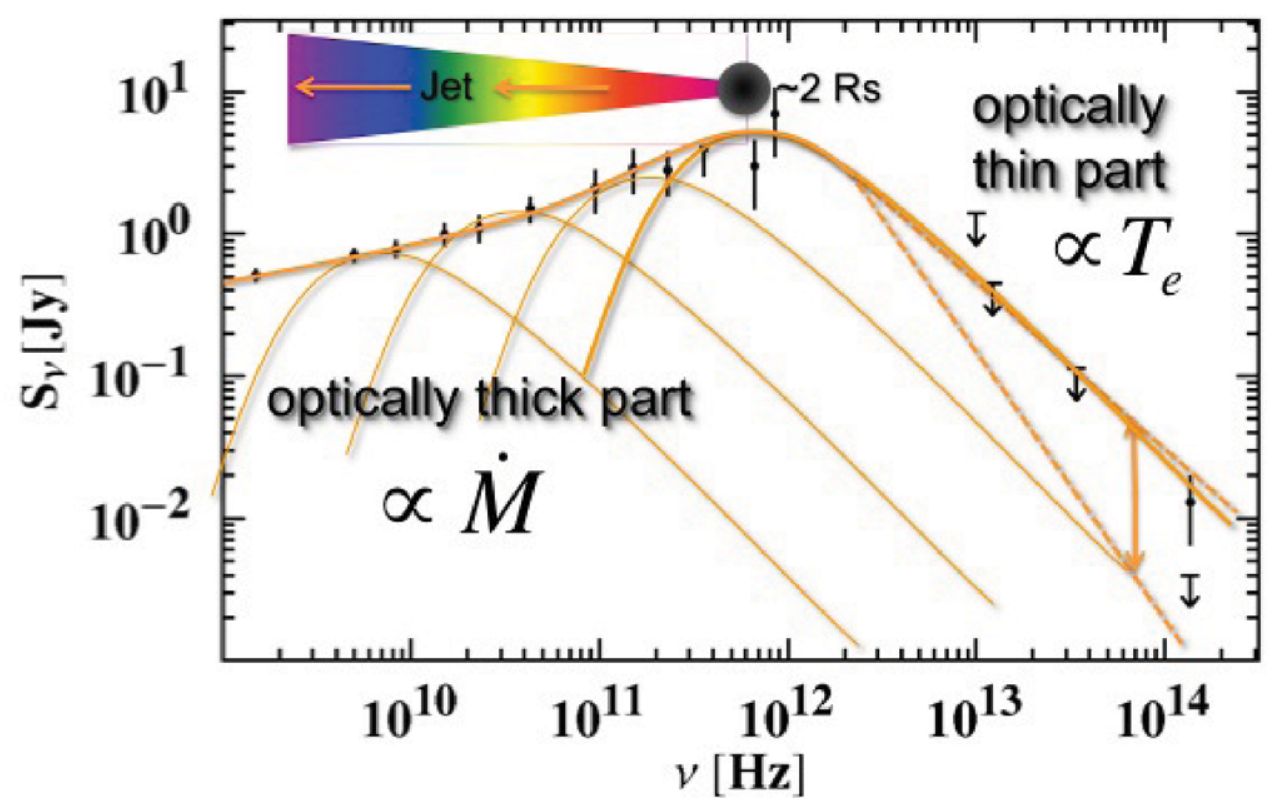

Figure 2. Spectrum of Sgr A* and schematic overview of various contributions from a jet.

physical background, how many of the observed properties arise quite naturally in a jet model. Figure 2 shows the radio spectrum of Sgr $\mathrm{A}^{*}$ and a schematic view of the various jet model components. Assume a hot magnetized plasma is ejected close to the black hole from a compact nozzle and expands freely thereafter. The plasma in the nozzle will emit synchrotron radiation peaking between $100 \mathrm{GHz}$ and a few $\mathrm{THz}$ due to optical depth effects (almost black body radiation), producing the "submm-bump". If the electron distribution is non-Maxwellian, i.e., has a high-energy power-law tail, optically thin synchrotron radiation will appear, that can easily extend into the near-infrared or even X-ray regime (which could also have a contribution from inverse Compton emission from the submm-bump).

When the plasma leaves the nozzle and expands, it will expand with sound speed and advance with the bulk flow speed, roughly filling a conical Mach cone. Like in a multi-color accretion disk, the synchrotron emission will then peak at lower and lower frequencies the further out the plasma gets. This will naturally lead to an optically thick, flat radio spectrum and a source size $\propto \nu^{-1}$ (Blandford \& Königl 1979; Falcke \& Biermann 1995). With the jet-disk scaling mentioned above one can then readily explain flux and size of Sgr A* to zeroth order (see Falcke 2003, for a text-book level description). However, a simple Blandford-Königl model is not really appropriate, since close to the black hole the pressure gradient - and likely other processes as well - will lead to an acceleration of the jet, yielding an inverted spectrum and a steeper than $\nu^{-1}$ frequency-size relation (Falcke 1996; Falcke \& Markoff 2000).

To understand the variability behavior one has to remember that the radio/submm spectrum $(i 1 \mathrm{THz})$ is optically thick and thus originates in a superposition of emission components from different spatial regions, peaking at increasingly lower frequencies as their characteristic scale increases. The NIR/X-ray spectrum, on the other hand, is optically thin and comes from one, i.e., the smallest, spatial scale. Already Markoff et al. (2001) showed that the jet radio emission will mainly respond to changes in the density (and hence accretion rate, given the assumed jet-disk coupling), while the NIR/X-ray 
emission will be highly sensitive to changes in the electron temperature or power-law distribution. Comparing Sgr A* to a lightning storm on earth, one might say that the radio would reflect the bulk motion of the clouds and the winds, while NIR/X-rays are more like lightning within the clouds.

This explains the high NIR/X-ray variability and its tight correlation - NIR and X-ray both reflect co-spatial changes in the population of high-energy electrons in the nozzle region, close to the event horizon. Compared to the bulk density of the flow, the number of high-energy electrons is small and their cooling time is fast. Hence, already a small change in the efficiency of accelerating non-thermal electrons can lead to large changes in NIR and X-rays without affecting the overall energy budget too much.

On the other hand, to produce radio flares a major change in the bulk density of lowenergy electrons is required, i.e., a change in accretion rate and total jet power. When induced at the foot point the density surge will have to propagate with the flow speed outwards (see Falcke et al. 2009; Maitra et al. 2009, for detailed modeling). This suggests lower relative flux changes compared to NIR, time lags in the sense that higher-frequencies lead lower ones, and not necessarily a tight connection between major radio/mm-flares on one hand and NIR/X-ray-flares on the other. Of course, it is conceivable that a sudden increase in accretion rate onto the black hole, which is seen as a radio flare, will also lead to more efficient dissipation and particle acceleration. However, in any case NIR/radio delays are likely not easy to interpret and should certainly not be used to estimate, e.g., time scales for adiabatic expansion. On the other hand, time lags within the radio/submm-wave regime could have high diagnostic powers, since they are directly related to the flow speed. Hence, VLBI size and radio monitoring data combined would provide unique information about the acceleration and formation of astrophysical jets and in particular the longitudinal velocity profile.

\section{MHD modeling and event horizon imaging}

Parallel to the semi-analytic modeling a new breed of MHD models has become available, some of which are now geared towards Sgr A*. These models basically start from first principles following the evolution of a hot magnetized plasma as it is accreted onto a black hole. Initially, jets did not form naturally in these simulations (Stone et al. 1996; Hawley 2000), especially when the initial configuration contained only a toroidal magnetic field, while simulations with random or poloidal fields seemed well to be able to produce jet-like outflows (e.g., Koide et al. 1999; Meier et al. 2001). Nowadays, most MHD simulations include poloidal magnetic field and hence show some evidence for jetlike outflows. Given the excellent observational constraints on scales close to the event horizon, Sgr A* has now become a useful testbed also for numerical MHD simulations (e.g., Ohsuga et al. 2005; Noble et al. 2007; Chan et al. 2009; Mościbrodzka et al. 2009; Dexter et al. 2010; Hilburn et al. 2010).

For example, Mościbrodzka et al. (2009) have performed simulations based on an axisymmetric version of the GRMHD code harm, applying relativistic radiative transfer (grmonty: Dolence et al. 2009) and GR ray tracing (ibothros: Noble et al. 2007) during post-processing. These 2D simulations do indeed also show a jet-like outflow, but it appears rather faint. Compared to the early pure GR ray tracing simulations (Falcke et al. 2000) the shadow of the black hole remains visible and a significant part of the parameter range of the models can be excluded. In their simulations, Mościbrodzka et al. (2009) find that the VLBI sizes and other properties favor edge-on orientations and a fast spinning black hole. 


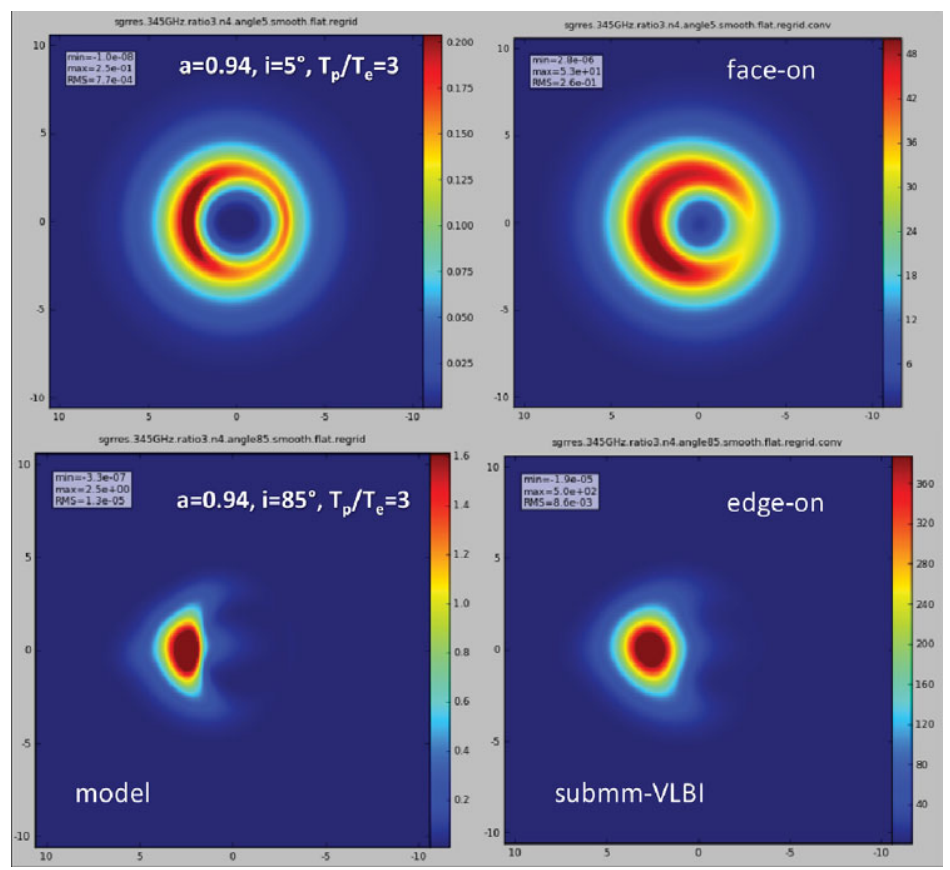

Figure 3. Left: Time-averaged appearance of 2D MHD accretion models at $345 \mathrm{GHz}$ (bottom-left: best-fit model) from Mościbrodzka et al. (2009). Right: Reconstructed images with a putative global submm-VLBI array. Top: face-on geometry, Bottom: edge-on geometry. The black hole shadow is clearly visible for face-one orientation but much less so for edge-on.

To demonstrate the potential of this approach, we have performed some simple simulations of future VLBI observations (Figure 3). For this we have taken the predicted intensity distributions at $345 \mathrm{GHz}$ (Fig. 3) from Mościbrodzka et al. (2009), smeared them with a Gaussian kernel according to the scattering law (taken from Falcke et al. 2009), and passed them through the interferometry simulation tool simdata2 in CASA (McMullin et al. 2007). Frequencies and baselines were appropriately scaled to avoid numerical problems and noise contributions were neglected. The array layout (Fig. 4) consisted of a number of existing (sub)mm-wave facilities (CARMA, SMT, JCMT), telescopes which are planned, are under construction or are not yet equipped for VLBI (LLAMA, ALMA, LMT, SPT), and one putative site in Peru to fill in missing baselines.

One can see that indeed such an array would be well matched to the resolution needed to study event-horizon scale structures in Sgr A*. High spin and inclination will produce a rather compact emission region with a size of $\sim 2-4 R_{\mathrm{g}}$ and a shadow that is difficult to see unless one achieves sufficient dynamic range $(\sim 100: 1)$.

It will be interesting to see how this picture changes as the simulations improve. Figure 4 (right) shows a snapshot of a single frame from a preliminary 3D GRMHD simulation with harm, again including ray tracing and radiation transport, for a black hole spin of $\mathrm{a}=0.9$. While in the $2 \mathrm{D}$ simulation the jet is very dim, to the point that it is not even visible in the emission maps presented here, the jet becomes much more prominent in the $3 \mathrm{D}$ case. This is apparently due to an increased electron temperature in the jet, possibly coming from increased acoustic heating. Clearly, the electron temperature in disk and jet plays and important role for the appearance of Sgr A*, but it is also the least understood parameter. Such simulations provide a taste for the future direction the field can evolve to. 

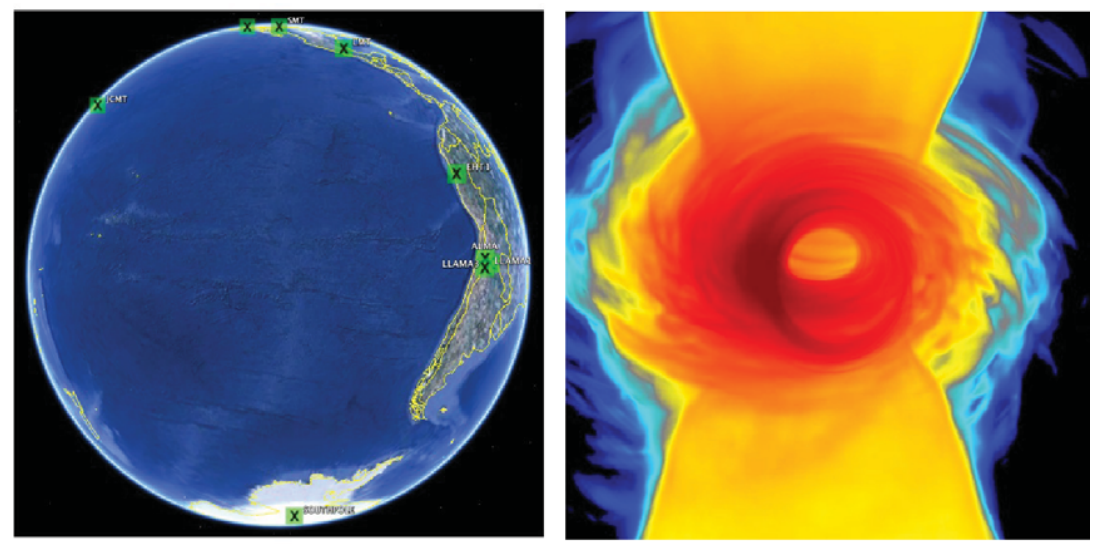

Figure 4. Left: Google-Earth view of the Earth with positions of the submm-VLBI stations used in the simulation as green boxes. Right: Snapshot of a 3D GRMHD simulation with similar parameters as in Fig. 3. Shown is the intensity at $345 \mathrm{GHz}$. The jet is much more visible, compared to the $2 \mathrm{D}$ case.

\section{Conclusions}

There is no other supermassive black hole with such a wealth of information. The properties are not different from many other radio cores in nearby low-power AGN when appropriately scaled to a very low accretion rate (Falcke \& Biermann 1999; Nagar et al. 2005; Markoff et al. 2008). In fact, within the fundamental plane of black hole activity (Merloni et al. 2003; Falcke et al. 2004; Körding et al. 2006), that has been discussed at length in this meeting, it represents almost an extreme "off-state".

Spectrum, size, and radio time-lags and evolution in Sgr A* strongly suggest a jetnature of the radio emission, with mildly relativistic outflow speeds. Since the the submmwave emission comes from the direct proximity of the event horizon, Sgr A* is an ideal source to image the shadow of the event horizon and to find out how astrophysical jets are formed - for example by combining mm-VLBI observations and time-lag measurements.

\section{References}

Aitken, D. K., Greaves, J., Chrysostomou, A., et al. 2000, ApJL, 534, L173

Baganoff, F. K., Bautz, M. W., Brandt, W. N., et al. 2001, Nature, 413, 45

Balick, B. \& Brown, R. L. 1974, ApJ, 194, 265

Blandford, R. D. \& Königl, A. 1979, ApJ, 232, 34

Bower, G. C., Backer, D. C., Zhao, J. H., Goss, M., \& Falcke, H. 1999, ApJ, 521, 582

Bower, G. C., Falcke, H., Herrnstein, R. M., et al. 2004, Science, 304, 704

Bower, G. C., Falcke, H., Wright, M. C., \& Backer, D. C. 2005, ApJL, 618, L29

Broderick, A. E. \& Loeb, A. 2006, MNRAS, 367, 905

Chan, C., Liu, S., Fryer, C. L., et al. 2009, ApJ, 701, 521

Coker, R., Melia, F., \& Falcke, H. 1999, ApJ, 523, 642

Dexter, J., Agol, E., Fragile, P. C., \& McKinney, J. C. 2010, ApJ, 717, 1092

Dodds-Eden, K., Porquet, D., Trap, G., et al. 2009, ApJ, 698, 676

Doeleman, S., Weintroub, J., Rogers, A. E. E., \& et al. 2008, Nature, 455, 78

Dolence, J. C., Gammie, C. F., Mościbrodzka, M., \& Leung, P. K. 2009, ApJs, 184, 387

Eckart, A., Baganoff, F. K., Morris, M. R., et al. 2009, A\&A, 500, 935

Eckart, A., Baganoff, F. K., Zamaninasab, M., et al. 2008, A\&A, 479, 625

Ekers, R. D., Goss, W. M., Schwarz, U. J., Downes, D., \& Rogstad, D. H. 1975, A $\& A, 43,159$ 
Falcke, H. 1996, ApJL, 464, L67

Falcke, H. 1999a, in ASP Conf. Ser. 186: The Central Parsecs of the Galaxy, ed. H. Falcke, A. Cotera, W. Duschl, F. Melia, \& M. J. Rieke (San Francisco: Astronomical Society of the Pacific), 148

Falcke, H. 1999b, in ASP Conf. Ser. 186: The Central Parsecs of the Galaxy, ed. H. Falcke, A. Cotera, W. Duschl, F. Melia, \& M. J. Rieke (San Francisco: Astronomical Society of the Pacific), 113

Falcke, H. 2003, Radio and X-ray emission from the Galactic black hole, ed. Falcke, H. \& Hehl, F. W. (IoP), 310-342

Falcke, H. \& Biermann, P. L. 1995, A\& A, 293, 665

Falcke, H. \& Biermann, P. L. 1999, A\&A, 342, 49

Falcke, H., Körding, E., \& Markoff, S. 2004, A\&SA, 414, 895

Falcke, H., Mannheim, K., \& Biermann, P. L. 1993, A\&A, 278, L1

Falcke, H. \& Markoff, S. 2000, A\& A, 362, 113

Falcke, H., Markoff, S., \& Bower, G. C. 2009, A\&A, 496, 77

Falcke, H. \& Melia, F. 1997, ApJ, 479, 740

Falcke, H., Melia, F., \& Agol, E. 2000, ApJL, 528, L13

Fish, V. L., Doeleman, S. S., Broderick, A. E., Loeb, A., \& Rogers, A. E. E. 2009, ApJ, 706, 1353

Genzel, R., Eisenhauer, F., \& Gillessen, S. 2010, ArXiv e-prints

Genzel, R., Schödel, R., Ott, T., et al. 2003, Nature, 425, 934

Ghez, A. M., Salim, S., Weinberg, N. N., et al. 2008, ApJ, 689, 1044

Gillessen, S., Eisenhauer, F., Fritz, T. K., et al. 2009, ApJL, 707, L114

Harko, T., Kovács, Z., \& Lobo, F. S. N. 2009, Class. Quant. Grav., 26, 215006

Hawley, J. F. 2000, ApJ, 528, 462

Herrnstein, R. M., Zhao, J.-H., Bower, G. C., \& Goss, W. M. 2004, AJ, 127, 3399

Hilburn, G., Liang, E., Liu, S., \& Li, H. 2010, MNRAS, 401, 1620

Johannsen, T. \& Psaltis, D. 2010, ApJ, 718, 446

Koide, S., Shibata, K., \& Kudoh, T. 1999, ApJ, 522, 727

Körding, E., Falcke, H., \& Corbel, S. 2006, A $\& A, 456,439$

Lynden-Bell, D. \& Rees, M. J. 1971, MNRAS, 152, 461

Maitra, D., Markoff, S., \& Falcke, H. 2009, AधA, 508, L13

Markoff, S., Bower, G. C., \& Falcke, H. 2007, MNRAS, 379, 1519

Markoff, S., Falcke, H., Yuan, F., \& Biermann, P. L. 2001, A\&A, 379, L13

Markoff, S., Nowak, M., Young, A., et al. 2008, ApJ, 681, 905

Marrone, D. P., Baganoff, F. K., Morris, M. R., et al. 2008, ApJ, 682, 373

Marrone, D. P., Moran, J. M., Zhao, J.-H., \& Rao, R. 2007, ApJL, 654, L57

Mauerhan, J. C., Morris, M., Walter, F., \& Baganoff, F. K. 2005, ApJL, 623, L25

McMullin, J. P., Waters, B., Schiebel, D., Young, W., \& Golap, K. 2007, in Astronomical Society of the Pacific Conference Series, Vol. 376, Astronomical Data Analysis Software and Systems XVI, ed. R. A. Shaw, F. Hill, \& D. J. Bell, 127-

Meier, D. L., Koide, S., \& Uchida, Y. 2001, Science, 291, 84

Melia, F. 1992, ApJL, 387, L25

Melia, F. \& Falcke, H. 2001, ARA\&A, 39, 309

Merloni, A., Heinz, S., \& di Matteo, T. 2003, MNRAS, 345, 1057

Meyer, L., Do, T., Ghez, A., et al. 2008, ApJL, 688, L17

Mościbrodzka, M., Gammie, C. F., Dolence, J. C., Shiokawa, H., \& Leung, P. K. 2009, ApJ, 706, 497

Nagar, N. M., Falcke, H., \& Wilson, A. S. 2005, A\& $A$, 435, 521

Narayan, R., Mahadevan, R., Grindlay, J. E., Popham, R. G., \& Gammie, C. 1998, ApJ, 492, 554

Noble, S. C., Leung, P. K., Gammie, C. F., \& Book, L. G. 2007, Class. Quant. Grav., 24, 259

Ohsuga, K., Kato, Y., \& Mineshige, S. 2005, ApJ, 627, 782

Porquet, D., Grosso, N., Predehl, P., et al. 2008, A\&A, 488, 549

Quataert, E. \& Gruzinov, A. 2000, ApJ, 539, 809 
Reynolds, S. P. \& McKee, C. F. 1980, ApJ, 239, 893

Schödel, R., Ott, T., Genzel, R., et al. 2002, Nature, 419, 694

Shen, Z.-Q., Lo, K. Y., Liang, M.-C., Ho, P. T. P., \& Zhao, J.-H. 2005, Nature, 438, 62

Stone, J. M., Hawley, J. F., Gammie, C. F., \& Balbus, S. A. 1996, ApJ, 463, 656

Yuan, F., Markoff, S., \& Falcke, H. 2002, A\& $A$, 383, 854

Yuan, Y., Cao, X., Huang, L., \& Shen, Z. 2009, ApJ, 699, 722

Yusef-Zadeh, F., Bushouse, H., Wardle, M., et al. 2009, ApJ, 706, 348

Yusef-Zadeh, F., Roberts, D., Wardle, M., Heinke, C. O., \& Bower, G. C. 2006, ApJ, 650, 189

Yusef-Zadeh, F., Wardle, M., Heinke, C., et al. 2008, ApJ, 682, 361

\section{Discussion}

FENDT: What is your heating model for the electrons? This is beyond MHD.

FALCKE: It is essentially local numerical dissipation.

RoDRIGUEz: Is there any chance of seeing the jet a few arcsec away from Sgr A* using an ultra-sensitive radio array like the EVLA?

FALCKE: Unfortunately does the Sgr A* size grow roughly with $\lambda$ while the scattering size grows with $\lambda^{2}$, hence the core will always be hidden at lower frequencies. Perhaps one could see some interaction region further out, but teh Galactic Center is a very complicated region.

Corbel: Do you have any idea why we live in a galaxy with the faintest LLAGN?

FALCKE: Estimates for the accretion rate based on environmental factors are all many orders of magnitude above the current value. Maybe we live in a special time. For example, the nearby supernova remnant Sgr A East has probably just swept over the black hole a few thousand years ago. Such local effects could easily disrupt a steady accretion flow.

DE Gouveia Dal Pino: Do you think that the time lag technique will be able to separate the complex motions that may occur at launching like, for instance, rotational velocity from the $v_{\mathrm{z}}$ component?

FALCKE: Good question - helical motion is indeed quite commonly observed in quasar jets with VLBI. Rotation will lead to differential beaming and hence will imprint a wobble as a function of frequency on top of the flare spectrum. Maybe with very sensitive broadband monitoring, e.g. with ALMA and SKA in the future, might such subtleties be detectable. 